\section{The unsung heroes}

\section{Peter Parham}

mmunology ostensibly began with ancient Greek physicians, who marvelled

at their observation that patients who had survived a bout of plague were resistant to the disease when it came around again. From this germ was born the idea of vaccination a small controlled infection, like a sort of immunological rehearsal, designed to confer future resistance to full-blown infection while causing only mere discomfort, rather than disease. What those Greeks also initiated was the tendency for immunologists to tackle their subject in a backward manner. They had become captivated by immunological memory, a late-acting feature of the immune response that only becomes useful if and when the first battle between person and pathogen - the primary infection has been won by other means.

Memory is the end point for adaptive immunity, the part of defence that is mediated by white blood cells called B cells and T cells. Although fascinating to immunologists for their strength and specificity, these soldiers are not speedy. Typically found in a state of suspended animation, B and T cells only become fit to fight about a week after combat begins. During this first week of a primary infection, defence is in the hands of innate immunity. One of several paths can be followed: the infection can gain the upper hand, leading to death or chronic, debilitating disease; alternatively, an acute infection can cause a temporary state of disease, which ends when innate immunity calls

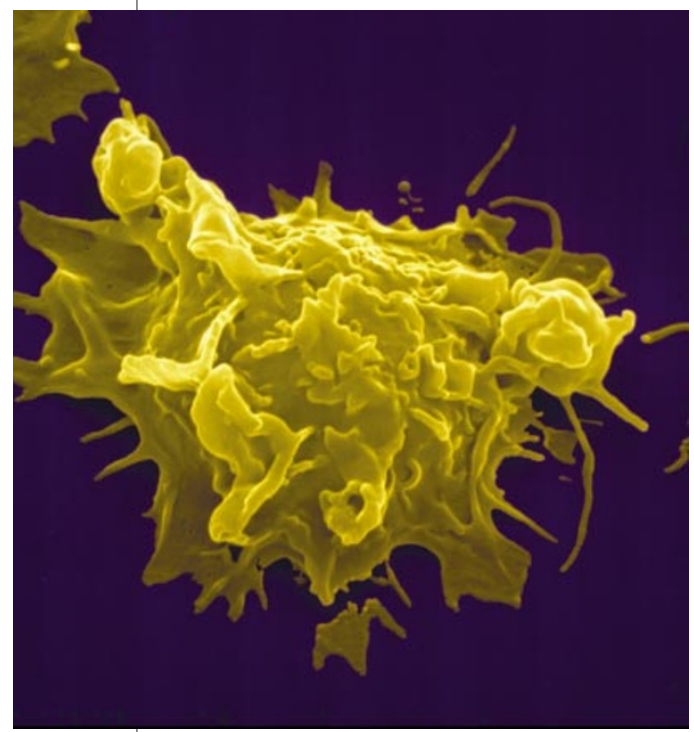

Attacking defence: a natural killer cell is among the first troops to enter immunological battle. up reinforcements in the guise of adaptive immunity. Patients who experience either of these outcomes have been carefully studied by physicians ever since the time of the ancient Greeks.

A third possibility is that the infection will be terminated quickly by innate immune mechanisms without the involvement of adaptive immunity and with no major symptom of disease. In this happy circumstance, in which health is maintained or only mildly perturbed, neither ancient Greeks nor their modern counterparts would be likely to consult a physician. Consequently, this most desirable of outcomes, in which the war is won with little collateral damage, remains understudied and poorly appreciated.

The fact that innate immune mechanisms can terminate infections should not be surprising - invertebrates and jawless fish survive infections solely on the wits of their innate immune systems. Even mutant laboratory mice with no adaptive immunity can survive and reproduce, as long as they are kept apart from their non-mutant brethren. Given this potential, what proportion of infections is terminated by innate immunity? In modern human society, we seem to endure intense and relentless exposure to all manner of infectious agents. Perhaps the very fact that most people are not perpetually sick is testament to innate immunity squelching most of the infections that we contract.

If we assume that innate immunity does, with some frequency, terminate infections before the onset of disease, there should also be natural selection for variants of proteins in the innate immune system that increase that frequency, even if only temporarily. Conversely, polymorphism among proteins of innate immunity is evidence of selection for such improvements in innate immunity. Several examples of this phenomenon have been revealed, such as the receptors and ligands that control the functions of natural killer (NK) cells, the lymphocytes of innate immunity.

NK cells are large, well-armed and circulate in a state of readiness, enabling them to enter and defend a tissue almost as soon as it becomes infected. NK cells kill infected cells and secrete cytokines, which rally the other cells of innate immunity, such as microbe-masticating macrophages. Moreover, through interaction with dendritic cells, NK cells help to decide if and when an adaptive immune response is needed. When innate immunity extinguishes an infection unaided, NK cells may kill off antigenbearing dendritic cells, preventing their migration to lymphoid tissues where they would otherwise activate T cells.

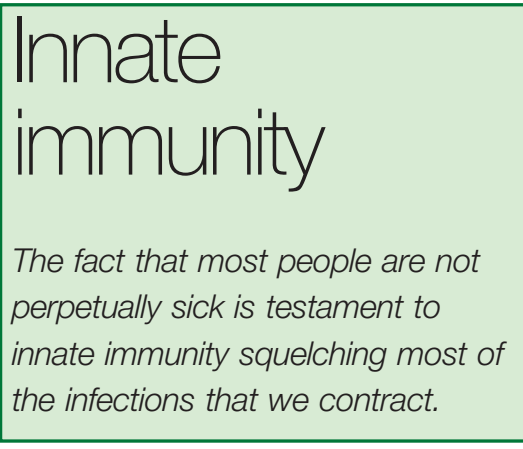

Unlike B and T cells, NK cells forego the process of rearranging genes to produce a repertoire of antigen receptors. Instead, they assemble different combinations of receptors from an impressive selection of activating and inhibitory cell-surface receptors. Many of these receptors bind specifically to major histocompatibility complex (MHC) class I or class I-like molecules - ligands that also control the activity of killer $\mathrm{T}$ cells and were previously thought to be dedicated to adaptive immunity. Prominent among receptors on NK cells are the killer-cell immunoglobulin-like receptors (KIRs), which, like their MHC class I ligands, are encoded by a diverse and rapidly evolving family of genes. People differ in the number and type of KIR genes that they inherit; the NK-cell repertoires of all but the closest of relatives are distinct. We are beginning to see how this individuality in NK-cell receptors is correlated with varying strength and weakness in the response of these cells to infection.

Innate immunity has sometimes been viewed as ancient in origin, and therefore highly optimized by natural selection. But does this mean that it is now set in evolutionary stone? Although the mechanisms of innate immunity predate those of adaptive immunity, it is not necessarily true that these mechanisms have stopped evolving. NK cells in humans and other species have many types of receptor, some conserved and others highly variable. These are not the properties of a highly optimized system, but rather of one that has been successively pushed in many different directions by natural selection. The fact that innate immunity is not unchanging, but rather is plastic and continuing to evolve, can be readily understood if we realize that innate immunity has the capacity to prevent primary infections from actually causing disease, an attractive feature that is out of bounds for adaptive immunity.

Peter Parham is at Stanford University School of Medicine, 300 Pasteur Drive, Stanford,

California 94305, USA.

\section{FURTHER READING}

Parham, P. (ed.) Immunol. Rev. 182, 1-289 (1999). Martin, M. P. et al. Nature Genet. 31, 429-434 (2002).

Scalzo, A. A. Trends Microbiol. 10, 470-474 (2002).

Moretta, A. Nature Rev. Immunol. 2, 957-964 (2002). 